\title{
DISTRIBUIÇÃO E DINÂMICA POPULACIONAL DO CASCUDINHO ALPHITOBIUS DIAPERINUS (COLEOPTERA: TENEBRIONIDAE) EM AVIÁRIOS DE FRANGO DE CORTE
}

\author{
D.H. Uemura ${ }^{2}$, L.F.A. Alves ${ }^{1 *}$, M.A.U. Opazo ${ }^{3}$, T.M. Alexandre ${ }^{2}$, D.G.P. Oliveira ${ }^{1}$, M.U. Ventura ${ }^{2}$
}

${ }^{1}$ Universidade Estadual do Oeste do Paraná, Centro de Ciências Biológicas e da Saúde, Laboratório de Zoologia de Invertebrados, Rua Universitária, 2069, CEP 85819-110, Cascavel, PR, Brasil. E-mail: lfaalves@unioeste.br

\section{RESUMO}

\begin{abstract}
Ocascudinho infesta cama de aviários eé uma das pragas-chave da avicultura moderna. Embora ineficiente, seu controle é baseado no uso de inseticidas químicos. Diante da falta de informações acerca da bioecologia do inseto nas condições do Brasil, realizou-se este trabalho em aviários comerciais de frangos de corte, em Cascavel, PR, sendo um aviário de piso de cimento e com alimentação automatizada (Cimento-Automático) e outro com piso de terra batida e comedouros pendulares (Terra-Manual), ambos tendo o piso coberto por cama de maravalha. As avaliações foram realizadas semanalmente com armadilhas colocadas sob comedouros (15 no aviário CimentoAutomático e 10 no Terra-Manual), sendo também registrada a temperatura da cama, nos locais de amostragem. Verificou-se uma tendência em se encontrar menor número de insetos no aviário Cimento-Automático, pela maior dificuldade do inseto em se desenvolver em locais com este tipo de piso. Maiores populações do inseto foram constatadas nas áreas de alojamento em função das elevadas temperaturas e na concentração da ração durante todo o período de criação.
\end{abstract}

PALAVRAS-CHAVE: Produção animal, manejo integrado de pragas, avicultura.

\section{ABSTRACT}

SPATIAL DISTRIBUTION AND POPULATION DYNAMICS OF THE LESSER MEALWORM, ALPHITOBIUS DIAPERINUS (COLEOPTERA: TENEBRIONIDAE), IN BROILER HOUSES. The lesser mealworm infests chicken-house litter and is a key pest in modern poultry raising. Its control is inefficient and done mostly using insecticides. Due the lack of information on pest bioecology in Brazil, this study was carried out in Cascavel, state of Paraná, Brazil, in two broiler houses: the first with concrete floor and automatic trough (concrete-automatic) and the second with compact soil floor and manual trough (manual-soil), both covered with wood residues litter. The assessments were made in the houses weekly using traps (15 in the concrete-automatic and 10 in the manual-soil). Temperature was also recorded. In general, there was a tendency to find a lower number of insects in the concrete-automatic house due to difficulty of insect development on this kind of floor. Greater populations were found in the shelter areas due to greater temperature and food concentration throughout the entire period of rearing.

KEY WORDS: Pest management, animal production, aviculture.

\section{INTRODUÇÃO}

O cascudinho, Alphitobius diaperinus (Panzer, 1797), é um inseto comum nos aviários de corte, onde condições favoráveis ao seu desenvolvimento, como temperatura alta, abrigo e alimento abundante favorecem o desenvolvimento de populações muito elevadas (KHAnet al., 1998). Durante a fase larval, vivem em meio à cama, buscando o solo para o desenvolvimento da fase pupal eoviposição. Estecomportamen- to permite a sobrevivência dos insetos na retirada da cama e garante a possibilidade de novos indivíduos reinfestarem o aviário após a colocação do novo substrato e/ou lote de aves (ARENDS, 1987; Gedenetal., 1987).

O cascudinho é ingerido pelas aves, no lugar da ração balanceada, reduzindo a ingestão denutrientes necessários afetando o desenvolvimento das aves (Despins; Axtel, 1994, 1995). Adultos e larvas perfuram a pele das aves, se alimentando do exsudato

\footnotetext{
${ }^{2}$ Universidade Estadual de Londrina, Departamento de Agronomia, Londrina, PR, Brasil.

${ }^{3}$ Universidade Estadual do Oeste do Paraná, Centro de Ciências Exatas e Tecnológicas, Cascavel, PR, Brasil.

*Bolsista de Produtividade em Pesquisa/CNPq.
} 
sanguíneo e também provocam ferimento no trato digestivo (papo e moela), podendo levar à morte pintinhos recém-eclodidos ou confinados em pequenos espaços. Além disso, podem contaminar a carcaça durante o processamento no abatedouro, quando se faz a extração do papo e da moela (CHERNAKI-LEFFER, 2004). Este inseto é também um reservatório e potencial transmissor de protozoários, platelmintos, fungos, bactérias, vírus patogênicos às aves, incluindo coronavírus (WATSON et al., 2000; CHERNAKI-LEFFER et al., 2002; BATES et al., 2004; SKOV et al., 2004; STROTHER; SteElman, 2001; VitTORI et al. 2007).

Conhecer a distribuição espacial deinsetos-praga é fundamental para estabelecer uma metodologia de controle adequada e auxiliar na tática de manejo em locais de grande densidade populacional, reduzindo a quantidade de inseticida utilizado. Neste sentido, trabalhos envolvendo a dinâmica populacional do cascudinho incluindo a distribuição espacial são escassos, sendo estes realizados em piso de terra batida e também em aviários de galinhas poedeiras (Fernandes et al., 1995; Strother; SteElman, 2001; Chernaki-Leffer, 2004; Pinto et al., 2005).

Considerando que informações do desenvolvimento do inseto, nestas duas condições encontradas nos aviários comerciais no Brasil, são importantes para se diferen-ciar o manejo, estudou-se a dinâmica populacional e distribuição de $A$. diaperinus em aviários de frango de corte com sistemas diferenciados de criação.

\section{MATERIAL E MÉTODOS}

Oestudo foi realizado em dois aviários comerciais localizados em Cascavel, PR (781 m dealtitude; $24^{\circ} 58^{\prime}$ S; $\left.53^{\circ} 26^{\prime} \mathrm{W}-\mathrm{GR}\right)$, no período de fevereiro de 2002 a março de 2004. Estes aviários são representativos dos sistemas de produção adotados na região oeste do Paraná.

Um dos aviários possuía $1.560 \mathrm{~m}^{2}(130 \times 12 \mathrm{~m})$, piso de cimento e com bebedouros e comedouros automáticos (Cimento-Automático), abrigando em média 21.000 aves por lote. O outro aviário media 720 $\mathrm{m}^{2}(60 \times 12 \mathrm{~m})$ e seu piso era de terra batida, alojando cerca de 6.500 aves, alimentadas com comedouros abastecidos manualmente com bebedouros automáticos (Terra-Manual), sendo que em ambos o piso era coberto por maravalha.

A amostragem populacional foi realizada por meio dearmadilhas modelo modificado por Arends (SAFRIT; AxTell, 1984), que consistia de um pedaço de papelão corrugado (20 cm de comprimento $30 \mathrm{~cm}$ delargura), enrolado e acondicionado em canos de PVC $(24 \mathrm{~cm}$ comprimento $\times 4 \mathrm{~cm}$ de diâmetro), sendo 15 para o aviário Cimento-Automático e dez no aviário TerraManual.
As armadilhas foram distribuídas nos aviários e instaladas sob os comedouros e semanalmente, elas foram substituídas. As armadilhas retiradas foram individualizadas em sacos plásticos lacrados e identificados, e armazenadas a $-4^{\circ} \mathrm{C}$, durante 24 horas para posterior contagem dos insetos. No início de desenvolvimento das aves, a da área no aviário de Cimento-Automático e $1 / 2$ do Terra-Manual era usado como "alojamento", onde as aves foram agrupadas. Após este período, as aves se dispersam por todo o aviário. Isto permite comparar as populações das áreas de alojamento e no restante do aviário.

Durante a troca das armadilhas mediu-se a temperatura da cama junto à armadilha e também a temperatura no interior dos aviários com um termômetro digital. A temperatura média do ambiente externo foi obtida junto ao Simepar.

Foram acompanhados três ciclos de utilização das camas nos dois aviários, sendo que no aviário Cimento-Automático foram coletados dados de quatro lotes na cama 1 , seis lotes na cama 2 e quatro lotes na cama 3. No aviário Terra-Manual foram coletados dados de seis, dois e cinco lotes, respectivamente, nas três camas, sendo adotado o delineamento estatístico de parcelas subdivididas e os dados foram analisados quanto à variância (teste F) e as médias populacionais comparadas pelo teste de Tukey ( $p<0,05)$ utilizando-se do programa estatístico Sisvar (FERREIRA, 2003).

\section{RESULTADOS E DISCUSSÃO}

\section{Aviário Cimento-Automático}

Flutuação populacional: foram realizadas 62 coletas durante os meses de fevereiro de 2002 a março de 2004, sendo que o número de larvas variou de 0 a 6.300 e de adultos foi de 0 a 828 insetos/armadilha. Além disso, o número médio de larvas coletadas sempre excedeu o número médio de adultos, ocorrendo poucas avaliações em que as armadilhas capturaram número de adultos superior ao de larvas (Tabela 1).

Da mesma forma, verificou-se em aviários na Carolina do Norte (EUA) a grande quantidade de larvas e adultos do cascudinho, sendo também o número de larvas maior que o número de adultos (PFeiffer;Axtell, 1980).

Ressalta-se que tal observação reflete a situação de contínua atividade reprodutiva do inseto, por este se encontrar sempre nas condições ideais para seu desenvolvimento e reprodução (CHERnAKI; AlmeIdA, 2001). Além disso, os adultos, ao contrário das larvas, podem também ser encontrados em grande número em outros locais abrigados além da cama, como frestas, madeira amontoada, solo etc. 
Verificou-seainda que, na primeira cama, o número médio de insetos nas armadilhas no primeiro lote foi de 385,4 larvas e 24,5 para adultos, e no lote seguinte, passou para 615,3 larvas e 208,7 adultos. No terceiro lote, houve tendência de estabilização da população (651,3 larvas e 248 adultos). No quarto lote houve uma aparente redução para 422 larvas e 160,2 adultos por armadilha.

Na cama 2, o número subiu de 233,7 larvas e 82,1 adultos para 298,1 larvas e 114,1adultos por armadilha. Na seqüência, no lote 3 e 4 houve estabilização da população. O lote 5 apresentou crescimento para 561,6 larvas e 239,2 adultos por armadilha. Noúltimo lote desta cama ocorreu queda expressiva de insetos provavelmente pelo menor período em que as aves permaneceram no aviário (apenas 10 dias com aves alojadas e as armadilhas instaladas).

$\mathrm{Na}$ última cama houve aumento no número de larvas na seqüência dos lotes, começando com 276,8 e 106,8 e terminando com 408,6 e 55,6 larvas e adultos, respectivamente, por armadilha.

Ressalta-se que o número relativamente baixo de insetos no início de cada ciclo da cama 1 para a cama 2 foi atribuído à retirada da cama usada, lavagem do aviário (piso, colunas, cortinas plásticas e paredes) e aplicação de inseticida antes da colocação da cama nova e entrada das aves.

Contudo, o manejo do aviário não determinou grandes alterações na flutuação populacional, sendo que, provavelmente, o fato das larvas bem como os adultos migrarem para o solo permite a sobrevivência dos insetos na retirada da cama e garante quantidade de indivíduos suficiente para infestar o aviário após a colocação do novo substrato e/ou lote de aves. Dessa forma, gradativamente a população aumenta a cada lote de aves que utiliza a mesma cama (ARENDS, 1987; GedEN, 1989).

Em relação à temperatura, verificou-se que a média na cama variou entre 25,3 (no lote 6 da cama 2) e $30,7^{\circ} \mathrm{C}$ (no lote 1 da primeira cama), sendo esta condição favorecida pelo acúmulo de cama que disponibiliza matéria-prima para a multiplicação de microrganismos saprofíticos.

Sabe-se que o período de incubação do ovo varia entre 8,9 e 3,0 dias na faixa de temperatura entre $22 \mathrm{e}$ $31^{\circ} \mathrm{C}$, respectivamente, e em temperaturas inferiores a $17^{\circ} \mathrm{C}$ não ocorre eclosão e que a fase larval dura em média de 33,2 dias a $28^{\circ} \mathrm{C}$ e 70,1 dias à temperatura de $22^{\circ} \mathrm{C}$ e o período pupal varia entre 9,7 e 4,0 dias nas temperaturas de $22 \mathrm{e} 31^{\circ} \mathrm{C}$, respectivamente (CHERNAKI; AlmeidA, 2001).

Assim, conclui-se que a temperatura no interior da cama e dos galpões de criação defrangos estão sempre em um patamar que possibilita o ótimo desenvolvimento do A. diaperinus. Além disso, considerando-se que o ciclo biológico de A. diaperinus de ovo a adulto ocorre em torno de 52 dias a $28^{\circ} \mathrm{C}$ e 31 dias a $31^{\circ} \mathrm{C}$ (CHERNAKI; Almeida, 2001), relacionando-se o número de insetos coletados ao acúmulo de camas, nas condições dos aviários estudados, forma-se uma geração e meia de insetos a cada lote, que ocorre a cada 45 a 50 dias.

Tabela 1 - Flutuação populacional de A. diaperinus no aviário Cimento-Automático (abril de 2002 a março de 2004).

\begin{tabular}{cclccc}
\hline Cama & Lote & \multicolumn{2}{c}{ Insetos coletados $^{1}$} & $\begin{array}{c}\text { Temperatura } \\
\text { da cama }\left({ }^{\circ} \mathrm{C}\right)^{2}\end{array}$ & $\begin{array}{c}\text { Temperatura } \\
\text { ambiente externa }\left({ }^{\circ} \mathrm{C}\right)^{3}\end{array}$ \\
\cline { 3 - 4 } & & Larvas & Adultos & $30,7 \pm 0,12$ & 24,0 \\
1 & 1 & $385,4 \pm 71.81$ & $24,5 \pm 3,45$ & $28,0 \pm 0,43$ & 18,5 \\
1 & 2 & $615,9 \pm 59,29$ & $208,7 \pm 48,74$ & $29,2 \pm 0,73$ & 15,6 \\
1 & 4 & $651,3 \pm 126,03$ & $248,0 \pm 0,73$ & $27,5 \pm 0,54$ & 18,6 \\
2 & 1 & $422,0 \pm 78,90$ & $160,2 \pm 32,71$ & $27,7 \pm 0,27$ & 22,3 \\
2 & 2 & $233,7 \pm 38,26$ & $82,1 \pm 10,98$ & $29,4 \pm 0,38$ & 23,7 \\
2 & 3 & $298,1 \pm 141,99$ & $114,1 \pm 22,48$ & $30,2 \pm 0,37$ & 21,5 \\
2 & 4 & $336,7 \pm 57,55$ & $162,8 \pm 44,01$ & $28,4 \pm 0,95$ & 17,1 \\
2 & 5 & $277,0 \pm 55,55$ & $117,1 \pm 23,98$ & $28,8 \pm 0,60$ & 17,3 \\
3 & 1 & $561,6 \pm 122,43$ & $239,2 \pm 54,38$ & $25,3 \pm 0,91$ & 18,4 \\
3 & 2 & $173,4 \pm 59,66$ & $18,0 \pm 5,04$ & $27,9 \pm 0,44$ & 21,9 \\
3 & 3 & $276,8 \pm 71,16$ & $106,8 \pm 28,72$ & $27,6 \pm 0,45$ & 22,4 \\
\hline
\end{tabular}

${ }^{1}$ Média $( \pm \mathrm{EP})$ de larvas e adultos por armadilha.

${ }^{2}$ Temperatura média $( \pm \mathrm{EP})$ tomada no momento da coleta.

${ }^{3}$ Temperatura média exterior do aviário. 
Tabela 2-Número médio de insetos coletados por armadilha no aviário Cimento-Automático. (abril de2002a março de2004).

\begin{tabular}{ccc}
\hline Cama & \multicolumn{2}{c}{ Média de insetos coletados nas armadilhas ${ }^{1}$} \\
\cline { 2 - 3 } & Região de alojamento & Região de crescimento $^{-}$ \\
\hline 1 & $826,0 \pm 61,44 \mathrm{~A}$ & $482,3 \pm 56,48 \mathrm{~B}$ \\
2 & $585,6 \pm 106,68 \mathrm{~A}$ & $276,0 \pm 89,59 \mathrm{~B}$ \\
3 & $454,8 \pm 118,60 \mathrm{~A}$ & $189,0 \pm 78,88 \mathrm{~B}$ \\
Média geral $^{2}$ & $622,1 \pm 95,57 \mathrm{~A}$ & $316,8 \pm 74,98 \mathrm{~B}$ \\
Temperatura $^{3}$ & $30,4 \pm 0,82 \mathrm{~A}$ & $26,8 \pm 0,69 \mathrm{~B}$ \\
\hline
\end{tabular}

${ }^{1}$ Médias $( \pm \mathrm{EP})$ seguidas pela mesma letra na linha não diferem entre si, pelo teste de Tukey $(\mathrm{P}<0,05)$.

${ }^{2}$ Número médio de insetos coletados nas armadilhas localizadas nas diferentes regiões.

${ }^{3}$ Temperatura média da cama $\left({ }^{\circ} \mathrm{C}\right)$.

Tabela 3 - Flutuação populacional de A. diaperinus no aviário Terra-Manual (fevereiro de 2002 a fevereiro de 2004).

\begin{tabular}{|c|c|c|c|c|c|}
\hline \multirow[t]{2}{*}{ Cama } & \multirow[t]{2}{*}{ Lote } & \multicolumn{2}{|c|}{ Insetos coletados $^{1}$} & \multirow{2}{*}{$\begin{array}{l}\text { Temperatura } \\
\text { da cama }\left({ }^{\circ} \mathrm{C}\right)^{2}\end{array}$} & \multirow{2}{*}{$\begin{array}{c}\text { Temperatura } \\
\text { ambiente externa }\left({ }^{\circ} \mathrm{C}\right)^{3}\end{array}$} \\
\hline & & Larvas & Adultos & & \\
\hline 1 & 1 & $370,1 \pm 93,48$ & $48,9 \pm 9,83$ & $26,1 \pm 1,71$ & 23,5 \\
\hline 1 & 2 & $765,3 \pm 98,76$ & $155,1 \pm 43,53$ & $29,2 \pm 0,73$ & 19,6 \\
\hline 1 & 3 & $926,3 \pm 233,74$ & $107,3 \pm 28,89$ & $30,2 \pm 1,37$ & 16,9 \\
\hline 1 & 4 & $621,1 \pm 138,92$ & $48,3 \pm 5,36$ & $30,3 \pm 1,08$ & 18,8 \\
\hline 1 & 5 & $650,5 \pm 83,14$ & $46,0 \pm 4,30$ & $31,4 \pm 0,62$ & 22,2 \\
\hline 1 & 6 & $195,2 \pm 33,47$ & $25,3 \pm 3,85$ & $32,4 \pm 0,49$ & 23,7 \\
\hline 2 & 1 & $306,6 \pm 93,94$ & $41,2 \pm 11,12$ & $30,3 \pm 0,48$ & 23,6 \\
\hline 2 & 2 & $428,1 \pm 98,87$ & $144,4 \pm 51,49$ & $29,2 \pm 0,65$ & 17,9 \\
\hline 3 & 1 & $585,6 \pm 161,61$ & $96,3 \pm 15,43$ & $25,0 \pm 0,36$ & 16,3 \\
\hline 3 & 2 & $1207,9 \pm 292,18$ & $170,3 \pm 23,38$ & $25,8 \pm 0,82$ & 19,4 \\
\hline 3 & 3 & $1379,6 \pm 228,30$ & $123,0 \pm 20,44$ & $29,5 \pm 0,58$ & 22,5 \\
\hline 3 & 4 & $379,7 \pm 62,47$ & $82,7 \pm 15,94$ & $31,0 \pm 0,69$ & 22,4 \\
\hline 3 & 5 & $1149,6 \pm 120,87$ & $76,1 \pm 11,69$ & $33,4 \pm 0,42$ & 23,4 \\
\hline
\end{tabular}

${ }^{1}$ Média $( \pm \mathrm{EP})$ de larvas e adultos por armadilha.

${ }^{2}$ Temperatura média $( \pm \mathrm{EP})$ tomada no momento da coleta.

${ }^{3}$ Temperatura média do ambiente externo obtida pelo Simepar.

Tabela 4 - Média de insetos coletados por armadilha no aviário Terra-Manual (fevereiro de 2002 a fevereiro de 2004).

\begin{tabular}{ccc}
\hline Cama & \multicolumn{2}{c}{$\begin{array}{c}\text { Média de insetos } \\
\text { coletados nas armadilhas }\end{array}$} \\
\cline { 2 - 3 } & $\begin{array}{c}\text { Região de } \\
\text { alojamento }\end{array}$ & $\begin{array}{c}\text { Região de } \\
\text { crescimento }\end{array}$ \\
\hline 1 & $962,5 \pm 59,00 \mathrm{~A}$ & $357,3 \pm 61,88 \mathrm{~B}$ \\
2 & $554,5 \pm 97,64 \mathrm{~A}$ & $365,8 \pm 104,90 \mathrm{~A}$ \\
3 & $1276,2 \pm 78,05 \mathrm{~A}$ & $812,1 \pm 67,01 \mathrm{~A}$ \\
№ de insetos & $931,1 \pm 78,23 \mathrm{~A}$ & $516,7 \pm 107,63 \mathrm{~B}$ \\
Temperatura $^{3}$ & $31,7 \pm 0,61 \mathrm{~A}$ & $27,4 \pm 0,57 \mathrm{~B}$ \\
\hline
\end{tabular}

${ }^{1}$ Médias $( \pm E P)$ seguidas pela mesma letra na linha não diferem entre si, pelo teste de Tukey $(\mathrm{P}<0,05)$.

${ }^{2} \mathrm{Número}$ médio de insetos coletados nas armadilhas localizadas nas diferentes regiões.

${ }^{3}$ Temperatura média da cama $\left({ }^{\circ} \mathrm{C}\right)$.
Tabela 5 - Número médio de insetos coletados nas armadilhas em dois aviários (fevereiro de 2002 a março de 2004).

\begin{tabular}{lc}
\hline Aviário (tipo de piso) & $\begin{array}{c}\text { Média total de } \\
\text { insetos/armadilha }\end{array}$ \\
\hline Cimento- Automático & $434,9 \pm 83,29 \mathrm{~b}$ \\
Terra- Manual & $707,1 \pm 76,05 \mathrm{a}$ \\
\hline
\end{tabular}

${ }^{1}$ Médias ( \pm EP) seguidas pela mesma letra nas colunas não diferem entre si, no teste de Tukey $(\mathrm{P}<0,05)$.

SALIN et al. (2000) verificaram que a presença de insetos é mais significativa sob os comedouros onde a temperatura é maior se comparada com as proximidades da parede, estando diretamente relacionado com o número de insetos, com média de 180 e 22,2 larvas e adultos e 97,4 e 17,9 larvas e adultos, respectivamente, para as armadilhas instaladas sob os 
comedouros e nas proximidades da parede. Além disso, foi constatado que a temperatura do solo influenciou na preferência de lugares para a empupação durante o período de desenvolvimento do inseto, visto que sob os comedouros a temperatura do solo esteve na faixa de $30^{\circ} \mathrm{C}$, próximo às paredes, de $16^{\circ}$ $\mathrm{C}$, que corresponde aolimite mínimo para o desenvolvimento dos estágios imaturos.

Considerando o efeito de inseticidas, as aplicações nos aviários não foram regulares e a redução populacional foi pequena, haja vista que na cama 2, o número de larvas caiu de 16.682 para 13.003, e logo em seguida subiu para 26.682, mesmo com as sucessivas aplicações de inseticidas, realizadas no início do alojamento dos lotes 1, 2 e 3 dessa cama. Esta situação reflete a realidade da maioria dos aviários e, dentre as causas, as falhas na tecnologia de aplicação são as mais prováveis, já que os insetos, na ausência das aves ou de condições adequadas, desenvolveram mecanismos comportamentais adaptativos para exposição a baixas temperaturas, tais como migração para outros locais no aviário, isso pode ter dificultado seu controle nos intervalos de um lote para outro quando o galpão está sem aves e às vezes sem a cama também (RENAULT et al., 1999). Da mesma forma, os insetos podem ter desenvolvido resistência ao ingrediente ativo repetidamente utilizado (piretróide) conforme informações do produtor (CHERNAKI-LEFFER, 2004).

Distribuição espacial: durante as coletas foram observadas diferenças no número de insetos e de temperatura entre a região de alojamento ( $1 / 3$ da área total) e a região decrescimento das aves (2/3 restantes da área total), sendo a primeira localizada junto à entrada de ambos os aviários.

Nas três camas, o número de insetos na região de alojamento foi sempre maior que nas demais áreas, sendo que no total, a região de alojamento apresentou a média de 622,1 insetos/armadilha e a região de crescimento uma média de 316,8 insetos/armadilha.

Nota-se uma relação do número de insetos com a temperatura, pois locais com maiores números de insetos apresentam maior temperatura e menor número de insetos, menor temperatura (Tabela 2).

Este fato contraria observações de STROTHER; STEELMAN (2001), segundo os quais os besouros nem sempre se agregam em locais preditos, atribuindo como provável causa para tal fenômeno, o pequeno tamanho dos insetos que retarda a sua dispersão pelo aviário, em busca de locais mais apropriados para seu desenvolvimento, e que somente nas últimas semanas os besouros se agregam em tais áreas.

Da mesma forma, CHERNAKI-LEFFER(2004) não constatou nenhum tipo de relação entre o número de insetos e a temperatura nos diversos pontos de coleta.

Verificou-se também que na região de alojamento a temperatura média da cama foi significativamente superior em relação à outra região (respectivamente 30,4 e $26,8^{\circ} \mathrm{C}$ ), corroborando o estudo de CHERNAKILEFFER (2004), segundo o qual, armadilhas dispostas na região de alojamento também apresentaram temperatura significativamente mais alta.

A temperatura da cama na região onde as aves eram confinadas no início de cada lote apresentou-se elevada se comparada com a média do restante do aviário, provavelmente pelo fato de que neste local as aves permaneciam maior período de criação, fazendo com que a cama desta área a presentasse um acúmulo maior de excremento e ração ocorrendo fermentação, pelo aumento da profundidade da cama e elevando a temperatura do substrato, favorecido pelo calor das aves confinadas.

\section{Aviário Terra-Manual}

Flutuação populacional: no período de fevereiro de 2002 a fevereiro de 2004 foram realizadas 69 amostragens e, assim como no aviário Cimento-Automático, o número médio de larvas coletadas por lotes sempre excedeu o número médio deadultos (Tabela3).

A quantidade total de insetos coletados por armadilha variou de 0 a 8.978 larvas e de 0 a 2.621 adultos, com grande variação no número em todas as avaliações. Assim, na primeira cama que começou com 370,1 larvas e 48,9 adultos no lote um, subindo para 765,3 e 155,1 e se estabelecendo (926,3 e 107,3), caindo no lote quatro para 621,1 e 48,3 larvas e adultos, respectivamente. No lote cinco voltou a crescer, atingindo o número de 650,5 larvas e caindo no último para 195,2 larvas e 25,3 adultos. A queda no número de insetos é devido ao período de criação das aves que foi reduzido excepcionalmente neste último lote por exigência da integradora.

O baixo número de insetos no início de cada lote deve-se à retirada da cama e a limpeza do aviário como já citado no aviário anterior.

A média de temperatura da cama variou de $25^{\circ} \mathrm{C}$ no lote 1 da cama 3 a $33,4^{\circ} \mathrm{C}$ no último lote da cama 3 , não havendo relação entre a variação desta com a temperatura ambiente, confirmando observações feitas no aviário Cimento-Automático. Além disso, CHERNAKI-LEFFER (2004) relacionou esta variação diretamente com o acúmulo de cama pela sua reutilização.

Assim como foi constatado no aviário CimentoAutomático, as aplicações de inseticidas químicos também foram irregulares e ineficientes, realizadas previamente ao alojamento dos lotes 3,4 e 6 na cama 1 e lotes 2 e 3, da cama 2 . Na 1ạ cama, o número de insetos continuou alto apesar da utilização desta forma de controle. A segunda aplicação realizada no quinto lote obteve êxito parcial, causando grande redução no número de insetos coletados, e as outras aplicações não reduziram o número de insetos. 
Distribuição espacial: neste sistema de criação também foram observadas diferenças nos números de insetos na região de alojamento e na região de crescimento do galpão. A temperatura da região de alojamento apresentou a média de $31,7^{\circ} \mathrm{C}$, contrastando com a região de crescimento, em que a média ficou em $27,4^{\circ} \mathrm{C}$. As regiões de alojamento e crescimento apresentaram na primeira cama $32,7 \mathrm{e} 27,2^{\circ} \mathrm{C}$, na segunda foi de 31,6 e $27,9^{\circ} \mathrm{C}$ e na terceira 30,8 e $27^{\circ} \mathrm{C}$, respectivamente.

Onúmero de insetos coletados por armadilhas no primeiro ciclo de cama 1 foi de 962,5 na região de alojamento e de 357,3 na região de crescimento. Nas camas seqüenciais a região de alojamento abrigou 554,5 contra 365,8 na região de crescimento e $1.276,2$ contra 812,1 nas distintas regiões da terceira cama (Tabela 4). No total, verificou-se média de931,1 e516,7 insetos, respectivamente.

As temperaturas de cada região demonstraram variações significativas sendo de $31,7^{\circ} \mathrm{C}$ para a região de alojamento e $27,4^{\circ} \mathrm{C}$ para a região de crescimento, coincidindo com o aviário anterior.

A presença do piso de terra batida neste aviário pode ser o fator responsável pela similaridade do número de insetos coletados nas camas 2 e 3 , pois difere do aviário Cimento-Automático, onde o piso abaixo da cama é cimentado. Segundo Salin et al. (2000), a distribuição espacial de adultos está relacionada à alta umidade do solo, baixa compactação e densidade da superfície e, excetuando-se a umidade do solo, o mesmo ocorre com larvas e pupas.

Além disso, os dados aqui obtidos corroboram estudos realizados em aviário com piso não cimentado, onde a distribuição dos insetos foi relatada como sendo uniforme, sem relação de temperatura (Chernaki-Leffer, 2004), sendo também observado por Strother; Steelman (2001), conforme discussão anterior, que nem sempre os besouros se encontram agregados.

Comparação do número de insetos entre os aviários: apesar de ambos os galpões apresentarem condições favoráveis para o desenvolvimento do inseto, na comparação entre elas, em relação ao número de insetos coletados, verificou-se que o aviário CimentoAutomático apresentou uma quantidade significativamente menor (Tabela 5). Tal observação corrobora informações dos técnicos que prestam assistência aos produtores integrados.

Apesar de não haver estudos no sentindo de diferenciar sistemas de produção de aves pelo tipo de piso, a diferença na população observada no aviário com piso de terra $(707,1$ indivíduos) em relação ao aviário de cimento (434,9 indivíduos) deve-se provavelmente ao comportamento do inseto na fase larval em fazer galerias e empupar no solo
(Matias, 1992). É provável que o piso de cimento funcione como uma barreira, reduzindo locais adequados de abrigo que são fartamente encontrados no piso de terra batida.

Outro fator que pode ser apontado é relacionado ao sistema automatizado de alimentação do aviário Cimento-Automático, que além de ter a vantagem de ser abastecido automaticamente conforme o nível da ração em seu interior é reduzido, por ser ligado diretamente ao silo, oferece às aves a quantidade adequada de ração evitando desperdícios decorrentes do abastecimento manual feito por pessoal despreparado (Avisite, 2008). Neste caso, o excesso de ração, conforme comentado inicialmente, é um dos fatores que favorece o desenvolvimento de grandes populações do inseto nos aviários.

\section{AGRADECIMENTOS}

Ao CNPq pela concessão de bolsas de Iniciação Científica e de Produtividade, ao Simepar pelo fornecimento de dados meteorológicos, à Globoaves pelo auxílio financeiro e disponibilização dos aviários, e aos demais colegas de Laboratório de Zoologia de Invertebrados, pelo auxílio à pesquisa realizada.

\section{REFERÊNCIAS}

ARENDS, J.J. Control, management of the litter beetle. Poultry Digest, v.66, p.172-176, 1987.

FERREIRA, D.F. Sistema Sisvar para análises estatísticas. Disponível em: <http://www.dex.ufla.br/danielff/ dff02.htm>. Acesso em: 23 out. 2006.

AVISITE. Nutrição. Comedouros - tipos - vantagens e desvantagens. Disponível em: <http://www.avisite. com.br/cet/trabalhos.asp?codigo $=14>$. Acesso em: 12 nov. 2008.

BATES, C.; HIETT, K.L.; STERN, N.J. Relationship of Campylobacter isolated from poultry and from darkling beetles in New Zealand. Avian Diseases, v.48, p.138-147, 2004.

CHERNAKI, A.M.; ALMEIDA, L.M. Exigências térmicas, período de desenvolvimento e sobrevivência de imaturos de Alphitobius diaperinus (Panzer)

(Coleoptera: Tenebrionidae). Neotropical Entomology, v.30, p.365-368, 2001.

CHERNAKI-LEFFER, A.M.; BIESDORF, S.M.; ALMEIDA, W.V.B.; VIGNE, F. Isolamento de enterobactérias em Alphitobius diaperinus e na cama de aviários no oeste do Paraná, Brasil. Revista Brasileira de Ciência Avícola, v.4, p.243-247, 2002. 
CHERNAKI-LEFFER, A.M. Dinâmica populacional, estimativa da resistência a inseticidas e alternativas de controle para o cascudinho Alphitobius diaperinus (Panzer, 1797) (Coleoptera: Tenebrionidae). 2004. 123f. Tese (Doutorado em Ciências) - Departamento de Ciências Biológicas, Universidade Federal do Paraná, Curitiba, 2004.

DESPINS, J.L.; AXTELL, R.C. Feeding behavior and growth of Turkey poults fed larvae of the darkling beetle, Alphitobius diaperinus. Poultry Science, v.73, p.1526-1533, 1994.

DESPINS, J.L.; AXTELL, R.C. Feeding behavior and growth of broiler chicks fed larvae of the darkling beetle, Alphitobius diaperinus. Poultry Science, v.74, p. 331-336, 1995.

FERNANDES, M.A.; SANTOS, M.A.; LOMÔNACO, C. Ocorrência de artrópodos no esterco acumulado em uma granja de galinhas poedeiras. Neotropical Entomology, v.24, p.649-654, 1995.

FERREIRA, D.F. Sistema Sisvar para análises estatísticas . Disponível em: <http://www.dex.ufla.br/danielff/ dff02.htm>. Acesso em: 23 out. 2003.

GEDEN, C.J. Understanding and controlling litter beetles. Poultry Digest, v.68, p.142-144, 1989.

GEDEN, C.J.; EDWARDS, T.D.; ARENDS, J.J; AXTELL, R.C. Efficacies of mixtures of disinfectants and insecticides. Poultry House, v.66, p.659-665, 1987.

KHAN, B.A.; DAY, P.A.; GOONEWARDENE, L.A; ZUIDHOF, M.J.; HAWKINS, G. Efficacy of tetrachiorvinphos insecticide dust against darkling beetles in commercial broiler chicken barns. Canadian Journal of Animal Science, v.78, p.23-725, 1998.

MATIAS, R.S. Controle de Alphitobius diaperinus em piso e cama de aviários. Pesquisa Agropecuária Brasileira, v.27, p.205-207, 1992.

PFEIFFER, D.G.; AXTELL, R.C. Coleoptera of poultry in caged-layer houses in North Carolina. Environmental Entomology, v.9, p.21-28, 1980.

PINTO, D.M.; RIBEIRO, P.B.; BERNARDI, E. Flutuação populacional de Alphitobius diaperinus (Panzer, 1797) (Coleoptera: Tenebrionidae) capturados por armadilha do tipo sanduíche em granja avícola no Município de
Pelotas, RS. Arquivos do Instituto Biológico, São Paulo, v.72, n.2, p.199-203, 2005.

RENAULT, D.; SALIN, C.; VANNIER, G.; VERNON, P. Survival and chill-coma in the adult lesser mealworm, Alphitobius diaperinus (Coleoptera: Tenebrionidae), exposed to low temperatures. Journal of Thermal Biology, v.24, p.229-236, 1999.

SAFRIT R.D.; AXTELL, R.C. Evaluations of sampling methods for darkling beetles (Alphitobius diaperinus) in the litter of turkey and broiler houses. Poultry Science, v.63, p.2368-2375, 1984.

SALIN, C.; DELETTRE, Y.R.; CANNAVACCIUOLO, M.; VERSON, P. Spatial distribution of Alphitobius diaperinus (Panzer) (Coleoptera: Tenebrionidae) in the soil of a poultry house along a breeding cycle. European Journal of Soil Biology, v.36, p.107-115, 2000.

SKOV, M.N.; SPENCER, A.G.; HOLD, B.; PETERSON, L.; ANERBY, B.; CARSTENSEN, B.; MADSON, M. The role of litter beetles as potential reservoir for Salmonella enterica and Thamophilic Campylobacter spp. between broiler flocks. Avian Disease, v.48, p.9-18, 2004.

STROTHER, K.O.; C.D. STEELMAN. Spatial analysis of Alphitobius diaperinus (Coleoptera: Tenebrionidae) in broiler production facilities. Environmental Entomology, v.30, p.556-561, 2001.

STROTHER, K.O.; STEELMAN, C.D; GBUR, E.E.

Reservoir competence of lesser mealworm (Coleoptera: Tenebrionidae) for Campylobacter jejuni (Campylobacterales: Campylobacteraceae). Journal of Medical Entomology, v.42, p.42-47, 2005.

VITTORI J.; SCHOCKEN-ITURRINO, R.P.; TROVÓ, K.P.; RIBEIRO, C.A.M.: BARBOSA, G.G.; SOUZA, L.M.;

PIGATTO, C.P. Alphitobius diaperinus como veiculador de Clostridium perfingens em granjas avícolas do interior paulista-Brasil. Ciência Rural, v.37, p.894-896, 2007.

WATSON, D.W.; GUY, J.S.; STRINCHAM, S.M. Limited Transmission of Turkey Coronavirus in Yong Turkeys by Adult Alphitobius diaperinus (Coleoptera:

Tenebrionidae). Journal of Medical Entomology, v.37, p.480-483, 2000.

Recebido em 24/8/06

Aceito em 13/11/08 